immunochromatography for all students $(n=2,619)$, the stool antigen test for 677 students and serum antibody test for 830 students. Of these, $126(4.7 \%$ 126/2619) tested positive by urine-based immunochromatography, 23 (3.3\% 23/677) tested positive by stool antigen test, and $50(6.0 \%$ 50/830) tested positive by serum antibody test. 163 students were positive in the three primary tests (urine-based immunochromatography positive and/or stool antigen enzyme-linked immunosorbent assay positive and/or serum antibody test positive). Finally, 138 (5.2\% 138/2619) tested positive for $H$. pylori on ${ }^{13} \mathrm{C}$-UBT.

Conclusions The current prevalence of $H$. pylori infection among students was low even in an area of Japan with a high incidence of gastric cancer.

\section{IDDF2018-ABS-0070 THE NEW INSERTION METHOD OF THE TRANS-NASAL ILEUS TUBE, THE ANTERIOR BALLOON METHOD, WAS APPLICABLE FOR THE SMALL BOWEL OBSTRUCTION: A RETROSPECTIVE CHART REVIEW OF THE 135 PATIENTS}

${ }^{1}$ Daisuke Yamaguchi* ${ }^{1}$ Kei lkeda, ${ }^{1}$ Yuki Takeuchi, ${ }^{1}$ Rikako Kinoshita, ${ }^{1}$ Tomohito Morisaki, ${ }^{1}$ Keisuke Ario, ${ }^{1}$ Seiji Tsunada, ${ }^{2}$ Yasuhisa Sakata, ${ }^{2}$ Kazuma Fujimoto. ${ }^{1}$ Department of Gastroenterology, National Hospital Organization Ureshino Medical Center, Japan; ${ }^{2}$ Department of Internal Medicine, Saga Medical School, Japan

\subsection{6/gutjinl-2018-IDDFabstracts.97}

Background The gastrointestinal decompression is the initial and effective therapeutic approach for the patients with small bowel obstruction. Our previous pilot case report using the newly developed trans-nasal ileus tube, the anterior balloon method, achieved effective decompression to the small bowel obstruction (Yamaguchi D, et al. Dig Endosc. 2018;30:120121). The present pilot study was investigated the effectiveness of new insertion method of transnasal ileus tube for small bowel obstruction in around 100 patients.

Methods The anterior balloon method used the ileus tube of $300 \mathrm{~cm}$ length with two (anterior and posterior) balloons. After insertion of the tube into the duodenum, the anterior balloon was injected and suctioned repeatedly with $10 \mathrm{~mL}$ of air using the $10 \mathrm{~mL}$ syringe until the ileus tube reached closer to the obstruction (figure 1).

A total of 135 patients with small bowel obstruction treated from January 2011 to January 2018 were retrospectively reviewed. The patients were divided into two groups: those who used the new insertion method: anterior balloon method in 52 patients (ABM group) versus the ordinary insertion method in 82 patients (OIM group). The patient and causes of ileus; treatment outcomes; and adverse events were compared between the two groups.

Results The patient characteristics and symptoms on admission were not different between ABM and OIM group. Adhesive ileus was the main cause of ileus between two groups.

The ABM group experienced significantly shorter insertion time $(28.2 \pm 9.1$ vs. $33.5 \pm 13.0 \mathrm{~min} ; \mathrm{p}=0.008)$ and significantly longer length of insertion tube $(223.2 \pm 32.3$ vs. 157.4 $\pm 31.7 \mathrm{~cm} ; \mathrm{p}<0.001)$ compared with OIM group. And ABM group experienced significantly shorter time for relief of clinical symptoms. The mean duration of the overall insertion tube and restarting of the meals in ABM group were significantly shorter than in OIM group. There were no significant differences in adverse events between two groups.

Conclusions The anterior balloon method was shorter insertion time, longer length of the insertion tube and superior to the ordinary insertion method for improvement of clinical symptoms. The anterior balloon method is convenient and worthy to achieve decompression for patients with small bowel obstruction.

(a)

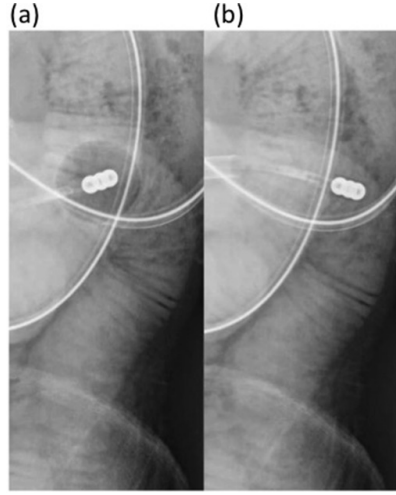

(c)

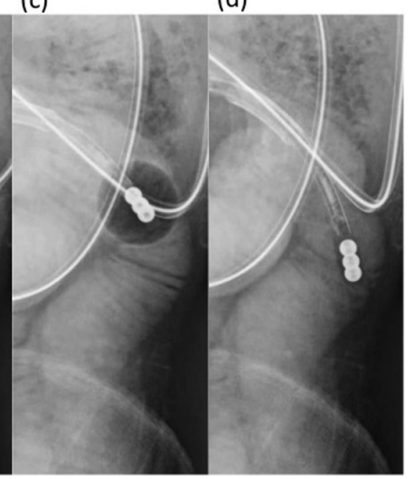

Abstract IDDF2018-ABS-0070 Figure 1 The anterior balloon method

\section{IDDF2018-ABS-0071 COMPARISON OF PROPOFOL SEDATION BETWEEN THE ENDOSCOPY ROOM AND OPERATION ROOM DURING ENDOSCOPIC SUBMUCOSAL DISSECTION FOR GASTRIC TUMOUR: A PROPENSITY SCORE MATCHING ANALYSIS}

'Daisuke Yamaguchi*, 'Yuki Takeuchi, 'Kei lkeda, 'Rikako Kinoshita, 'Tomohito Morisaki, ${ }^{1}$ Keisuke Ario, ${ }^{1}$ Seiji Tsunada, ${ }^{2}$ Naoko Yamaguchi, ${ }^{2}$ Ryo Katsuki, ${ }^{3}$ Naoyuki Tominaga, ${ }^{3}$ Shinichi Ogata, ${ }^{4}$ Yasuhisa Sakata, ${ }^{4}$ Kazuma Fujimoto. ${ }^{1}$ Department of Gastroenterology, National Hospital Organization Ureshino Medical Center, Japan; ${ }^{2}$ Department of Anesthesiology, National Hospital Organization Ureshino Medical Center, Japan; ${ }^{3}$ Department of Gastroenterology, Saga-Ken Medical Centre Koseikan, Japan; ${ }^{4}$ Department of Internal Medicine, Saga Medical School, Japan

\subsection{6/gutjnl-2018-IDDFabstracts.98}

Background Propofol, a short-acting sedative characterised by rapid recovery, has several advantages including induction of an appropriate sedation level and relative ease of safely maintaining this level. These advantages have led to a worldwide increase in the application of propofol for standard endoscopic procedures. Previous studies recommended the use of continuous propofol sedation for endoscopic submucosal dissection (ESD), but endoscopists must also give instructions for sedation while carrying out ESD procedures, because of the absence of the anesthesiologist in the endoscopy room in Japan.

The present study was performed to compare the safety of sedation using propofol during ESD in the endoscopy room versus the operation room.

Methods In total, 639 patients with gastric tumours who underwent ESD from January 2011 to August 2017 at Ureshino Medical Centre and Saga-Ken Medical Centre Koseikan were retrospectively reviewed. The patients were divided into two groups: those who underwent ESD in the endoscopy room (Group $\mathrm{E} ; \mathrm{n}=534$ ) versus operation room (Group O; $\mathrm{n}=105)$. Propensity score matching was used to compensate for the differences in age, sex, tumour size, procedure time, anaesthesia time and total propofol dose. The treatment outcome of ESD and the adverse events (desaturation, 
hypotension, bradycardia and arrhythmia) of sedation during ESD were evaluated between two groups.

Results Propensity score matching analysis created 91 matched pairs. Adjusted comparisons between Group E and O showed similar treatment outcomes of ESD (en bloc resection rate: $98.9 \%$ vs. $100 \%, \mathrm{p}=1.000$; perforation rate: $4.4 \%$ vs. $1.1 \%$, $\mathrm{p}=0.368$; delayed haemorrhage rate: $8.8 \%$ vs. $2.2 \%$, $\mathrm{p}=0.100)$. In adverse events, desaturation occurred significantly more often in Group E than O $(16.5 \%$ vs. 3.3\%, $p=0.005)$. There were no significant differences in other adverse events between the two groups (hypotension rate: $13.2 \%$ vs. $7.7 \%, \mathrm{p}=0.333$, bradycardia rate: $5.5 \%$ vs. $1.1 \%$, $\mathrm{p}=0.211$, and arrhythmia rate: $0 \%$ vs. $0 \%, \mathrm{p}=1.000$ ).

Conclusions Whereas a decrease in the desaturation rate was the only advantages of sedation with propofol in the operation room, sedation in the operation room might be required to ensure safer application of ESD for gastric tumours.

\section{IDDF2018-ABS-0075 MANAGEMENT OF ANAEMIA IN INFLAMMATORY BOWEL DISEASE}

Hang Hoi Michael Wong*, Ying Ki Shirley Tze. United Christian Hospital, Hong Kong

\subsection{6/gutjin-2018-IDDFabstracts.99}

Background Anaemia is the most common extraintestinal manifestation of IBD. It has a substantial impact on patients' morbidities and healthcare costs.

Methods We conducted a retrospective observational study on all our patients (total numbers: 224) suffering from IBD to determine whether anaemia was addressed during their clinic visits and whether treatment(s) were appropriately given. The haemoglobin, mean cell volume (MCV), ferritin, total iron binding capacity (TIBC), iron saturation (in\%) and CRP were reviewed.

Results Among the total 132 (59\%) Ulcerative colitis (UC) patients and 92 (41\%) Crohn's disease (CD) patients, 91 (40\%) patients suffered from anaemia at diagnosis. 93 (42\%) patients had ferritin, TIBC and iron saturation reviewed and documented. $71(32 \%)$ patients received iron supplements at some point since the diagnosis of IBD was made. All patients with iron deficiency anaemia were correctly identified and were offered iron supplements. However, of those suffered from iron deficiency anaemia, only 24 (34\%) patients had their ferritin, TIBC and iron saturation monitored every three months after adequate replacement to prevent recurrent iron deficiency. 25/91 (28\%) of IBD patients suffered from noniron deficiency anaemia (NIDA): 9 patients suffered from B12 deficiency and were all offered a parenteral replacement, 16 patients were given the diagnosis of anaemia of chronic disease (ACD), who demonstrated clinical or biochemical evidence of inflammation. During their most recent clinic visits, all patients had their haemoglobin reviewed and 47 (21\%) patients still suffered from anaemia. 10/47 (22\%) were noncompliant with their iron supplements; 16/47 (34\%) were due to active inflammation; the rest did not have a clear diagnosis.

Conclusions The aetiology of anaemia in IBD patients were mainly categorised into iron deficiency anaemia and NIDA. Adequate iron replacement and compliance are important in the treatment of iron deficiency. Recurrence of iron deficiency can be prevented by monitoring blood counts, ferritin, TIBC and iron saturation every three to six months. NIDA requires further work-up, and optimisation of IBD treatment should be first considered especially in ACD. In patients with persistent anaemia despite iron replacement and optimised IBD treatment, one should consider the use of erythropoiesis-stimulating agents.

\section{IDDF2018-ABS-0084 RECURRENT ACUTE PANCREATITIS IN A CHILD - AETIOLOGY BEING A RARE PANCREATIC DUCT ANOMALY}

Varun Palanati*. Narayana Medical College, Nellore, India

\subsection{6/gutjnl-2018-IDDFabstracts. 100}

Background Acute pancreatitis is an emerging problem in paediatrics. Although the cause is unclear, it may be explained by a heightened awareness of AP in children. Although magnetic resonance cholangiopancreatography (MRCP) is seldom required for the first attack of $\mathrm{AP}$, it constitutes a valuable tool in the evaluation of pancreaticobiliary abnormalities. Congenital anomalies and normal variants of the pancreatic duct are often detected as incidental findings in asymptomatic patients. Occasionally it produces symptoms in childhood and here is a patient presented to us with recurrent episodes of abdominal pain and MRCP helped us in diagnosing the condition and directed us for therapy.

Methods 13-year-old male presented to us with complaints of upper abdominal pain since 5 days, vomitings since 4 days. His abdominal pain is radiating to back. He had similar episodes for three times in the past 2 years but were treated conservatively at a local hospital and not been investigated. On examination, vitals were stable and abdominal examination was normal. In view of the history of recurrent acute pancreatitis, MRCP was done. It showed bifid duct of wirsung with duct of santorini opening into its anterior division with bulky body and tail of the pancreas. The patient was conservatively treated, and he recovered well

Results It was a case report showing pancreatic duct anomaly causing recurrent acute pancreatitis.

Conclusions Congenital variants of the biliopancreatic ductal system provide interesting challenges when discovered during the diagnostic workup of idiopathic acute recurrent pancreatitis. However, most of these variants are clinically irrelevant. Methods for the selection of patients most likely to benefit from invasive therapy need to be refined.

\section{IDDF2018-ABS-0086 NON-ALCOHOLIC FATTY PANCREAS DISEASE AS AN INDEPENDENT PREDICTOR OF ACUTE PANCREATITIS: A RETROSPECTIVE STUDY OF 264 PATIENTS}

${ }^{1}$ Jiarong Xie*, 'Lei Xu. 'Ningbo University, College of Medicine, Ningbo, China; Department of Gastroenterology, Ningbo First Hospital, Ningbo, China; ${ }^{2}$ Department of Gastroenterology, Ningbo First Hospital, Ningbo, China

\subsection{6/gutjnl-2018-IDDFabstracts. 101}

Background This study is aimed to investigate the relationship between non-alcoholic fatty pancreas disease (NAFPD) and the severity of acute pancreatitis (AP) and to assess the significance of pancreas attenuation in the prognosis and mortality of AP patients. 